\title{
Augmented Network Model For Engineering System Design
}

\author{
Gergana Bounova, Olivier de Weck \\ Massachusetts Institute of Technology \\ \{gergana,deweck\}@mit.edu
}

\subsection{Introduction - Motivation}

Models are usually domain specific and even traditionally strictly system-specific. Various modeling tools have sprung up to try to accommodate many systems simultaneously, such as state space and nonlinear dynamics models. As system complexity and data quantity creep up, attempts at a unified systems theory have become more rampant. Network theory is one of the fields that has been recently engaged in this effort, even termed «network science». Network theory tools exist in many domains, and have been used widely without consolidating efforts for at least 4050 years. In this paper, we examine the relevance, benefits and deficiencies of network representation and analysis for engineering systems, and at the end recommend alternatives.

The overall difficulty with applying simple network models to engineering systems is that often nodes and links or node relationships are not uniform and not transitive. In an acquaintance network, for example, the relation of knowing someone is reversible, thus the network is undirected. All nodes are uniform (even in modeling hierarchies, nodes are of the same type), and all links are the same. For representation purposes geometry or order is irrelevant. This is not the case for most engineering systems, where at any level of abstraction components are assembled or arranged in particular ways to work properly. Moreover, the nodes and links rarely can be put in the same category. These are hybrid networks: in other words, networks comprised of nodes (and maybe links) of different types.

For example, modeling the components of a vehicle, the parts (or subsystems) of an airplane or the states of a formation of flying vehicles, is not as simple as pointing out the nodes and the physical connections. Depending on the level of abstraction chosen, links can mean physical connections, like an electric connector, welded point, influence connections, like magnetic fields, chemical bonds or concentration levels, in fuel 
mixtures; abstract connections, such as a transportation route. Links and nodes can have different capacities and costs, maintenance routines, dynamics. Link existence can vary with time. All of these properties of real systems make simple graph representations inadequate for a useful model.

This does not mean that simple metrics from a pure graph model could not be useful for engineering analysis. The key in this type of modeling is i) picking the right level of abstraction, ii) encoding the right level of detail. We call this approach "augmented network modeling for engineering design".

Various fields have applied network modeling to engineering applications, such as operations research (supply chains), electrical engineering (circuit (controls) theory), and more recently systems engineering (new systems of systems approaches). Most have found good algorithms to solve particular problems but met overall difficulty in applying general models due to domain knowledge specificity. Some of the successful models are discrete-continuous state-space models and object-process networks for formation flight vehicle networks and space mission planning [Bounova 2005][Simmons 2005][Hybrid Lab].

In this paper, we propose a state-space-like augmented network model, with high-level simple abstract network description and deeper level of engineering detail description content.

We first give examples of real systems models, with their network description and brief discussion of simple network statistics. Then, we describe in detail one of the models and discuss the benefits and deficiencies of network representation and how it can be adjusted.

\subsection{Model - System Examples}

An augmented network model is an attempt to capture domain-specific knowledge and yet be able to extract and analyze higher-level network properties. This method does not claim to solve the modeling problems of all systems imaginable, and it does require hard additional work for application adaptation. However such a hybrid representation allows a general plug-in of many models to the same network analysis toolbox. To investigate the relevance of network models, we analyze five different systems, some of which biological, social and technological. Those are described briefly in the following sections.

\subsubsection{Journal Publication Network for the MIT Engineering System Division Community}

This is a social network example, consisting of 196 journals as nodes: two journals are connected if one faculty member publishes in both. All nodes and links are of the same type and all links are bidirectional by definition. The data is gathered by taking a poll and recording citations. Figure 1 shows the entire dataset with its giant component and isolated clusters. The general journal area/topic is also indicated for the different 
clusters. The clustering per topic confirms that every faculty member publishes in a certain area, as expected. The more heterogeneous giant component (labeled "core") indicates that many researchers that publish in a few fields thus leading to an interdisciplinary department. The most connected journal is Management Science.

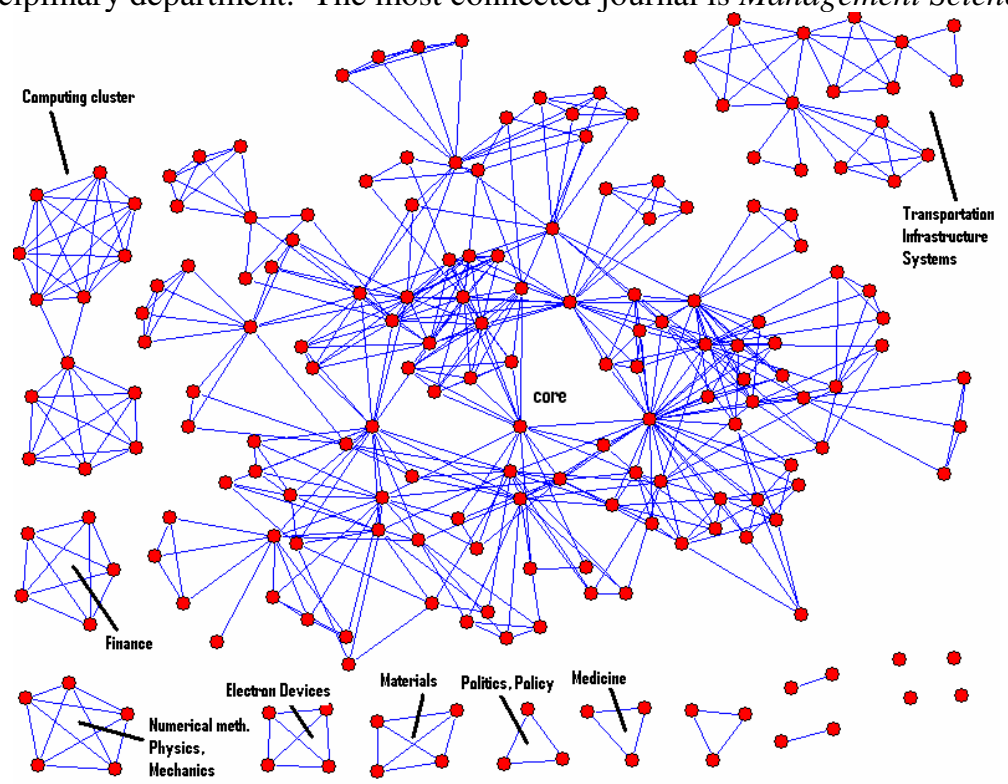

Figure 1: Journal publications network: two journals are connected if one faculty member publishes in both. 16 connected clusters are identified, as labeled.

\subsubsection{MAPK Reference Pathway Network}

The Mitogen-Activated Protein Kinase (MAPK) pathways transduce a large variety of external signals, leading to a wide range of cellular responses, including growth, differentiation, inflammation and apoptosis. In this biological network example, proteins are modeled as nodes and two proteins are connected if they interact. The data is experimentally verified, available from the KEGG database [KEGG dat]. Nodes are of the same type (but different molecules), links can vary depending on the type of interaction, such as activation or inhibition. This pathway was analyzed for three species, drosophila, yeast and human, include establishing structural similarity via coarse-graining and motif analysis. 


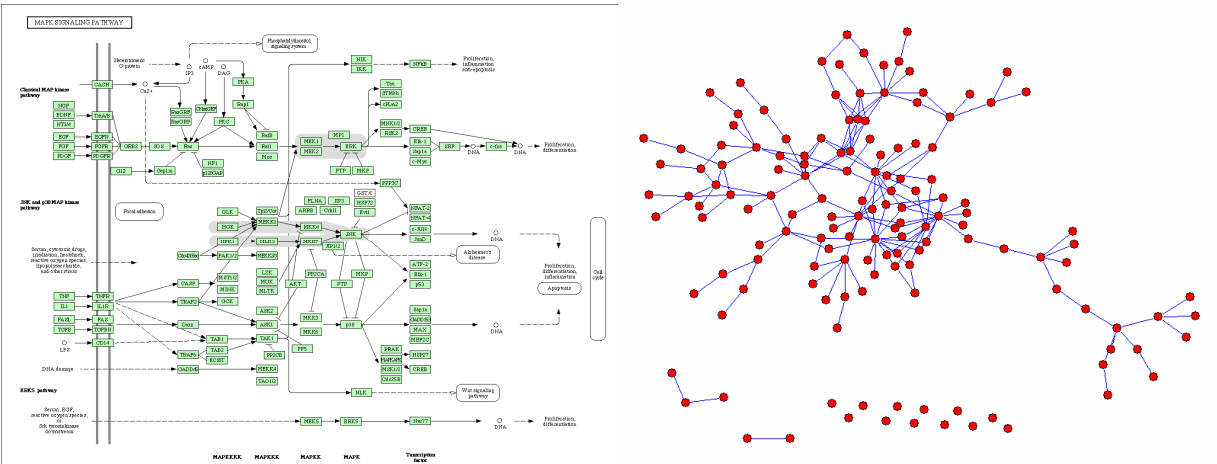

Figure 2: MAPK pathway representations: traditional representation (left) from KEGG database [KEGG dat] and Pajek representation of the same pathway.

\subsubsection{Car Frame Assembly Network}

The assembly network of passenger car frame components has been studied in the context of looking for multiple design alternatives for embedding flexibility into product components [Suh 2004]. In Figure 3 the rectangles represent individual components, i.e. nodes in the component network. The arcs represent physical connectivity between the components. The nodes are not identical: each one of them represents a uniquely different item, even though some nodes might be very similar, i.e. mirror images of each other due to symmetry of the vehicle. This is an example of a mechanical network in which geometry and relative position matters as much as pure connectivity for the proper operation of the system. Each component can be identified by its different name, position with respect to other components and different structure and function.
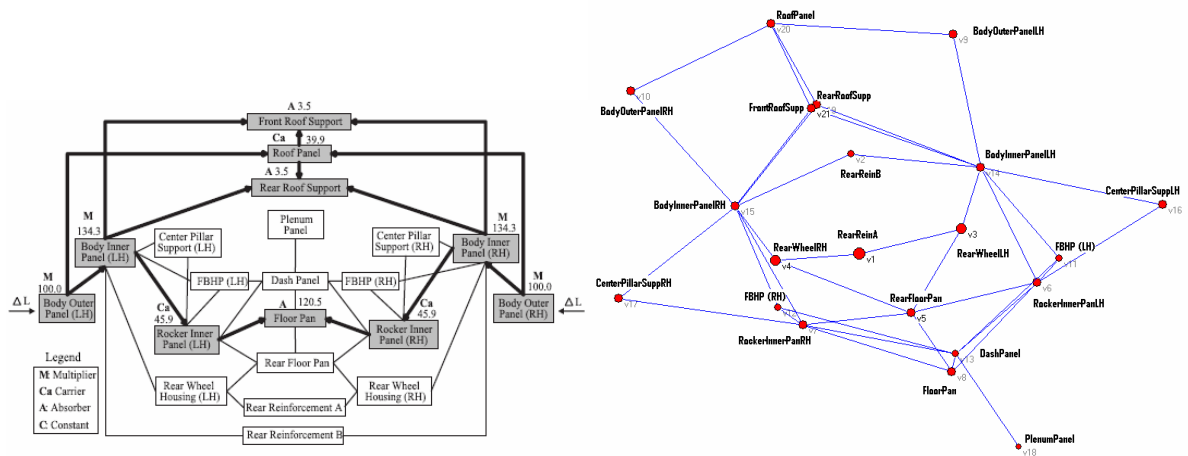

Figure 3: Car frame component network - wiring diagram (top) and Pajek plot (bottom). 3D location optimization algorithm preserves symmetry and relative positions. Right and left-hand sides are flipped in the Pajek plot. 


\subsubsection{Space Transportation Network Model}

There are states in space which a spacecraft occupy with minimal energy expense (ex. orbiting). State transitions require energy leaps, in the form of fuel burns, provided by the vehicle itself or an external force (another vehicle, by-passing a planet etc). Naturally, states can be modeled as nodes and transitions as links, so that the mission time-value is concentrated in the nodes, while the mission cost-energy spent is contained mainly in the links. This is not a perfect assumption, because transitions are not instantaneous and states are not cost-free (ex: stationkeeping and correction maneuvers). This representation without the inherent dynamics is shown in Figure 4. Here, neither all nodes, nor all links are of the same type. There are three types of nodes, surface, orbit and operational sequence nodes, and around 16 types of links, such as deorbit burn, orbit injection, landing and so on. This model was created with the purpose of generating and evaluating many architectures (scenarios) for lunar missions.
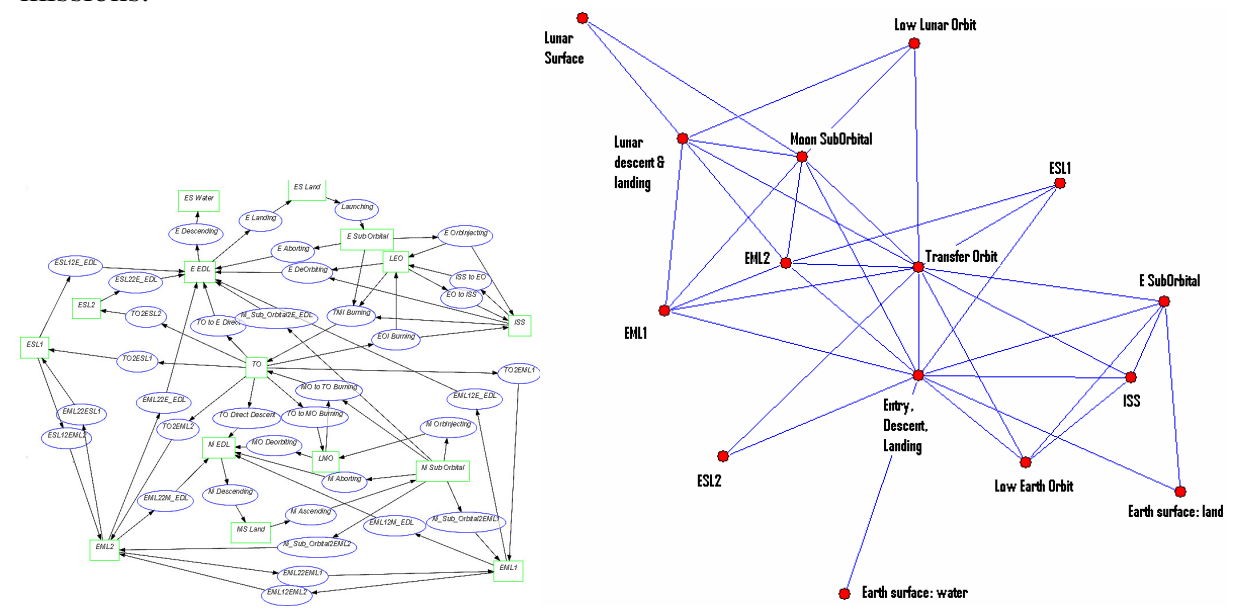

Figure 4: Space transportation network model for a lunar mission scenario. OPN (Object-Process Network, [Simmons 2005]) representation (left) and Pajek plot (right).

\subsection{Comparative Analysis, Network Statistics}

For all the systems discussed above, we discuss structure and dynamics analyzed with network modeling tools. Traditional network metrics, similar to the literature [Newman 2003], are shown in Table 1. Even from a coarse look, the simple network metrics in Table 1, show that even among technological networks, there is a great variety of directed versus undirected models, many versus few number of nodes, physical networks versus abstract (state transition) models. The average path length and diameter measures show the relative size of the network. For example, an average pathlength which is a small percentage of the number of nodes, is one of the characteristics of a small world. For the systems presented here, in general technological systems are not small worlds because of the effect of geometry and the importance of Euclidean 
distance. Degree correlations vary regardless of the type of system, sociological, biological or technological, contrary to claims that it should either positive or negative [Newman 2003]. Mean degree is smaller for technological systems in general, due to capacity, geometry and degrees of freedom constraints, but that varies from abstract to physical models. The space transportation network which is an abstract network, has a twice higher average nodal degree. Finally, all systems have different objectives for operations or performance measures.

A lot of network analysis concentrates on understanding system structure, modules, cohesiveness and hence critical components or nodes. Two structural experiments were done to gauge the relevance of these methods for technological systems. Figures 5 and 6 show the comparison of physically meaningful component breakdown versus the Newman-Girvan algorithm [Newman 2003] results for the car component network and the spacenet model. The top drawing in each figure shows the physically meaningful grouping of nodes. For example, on Figure 6, Earth and near-Earth nodes are grouped together. All transfer nodes together with Lagrange point orbits make another set. Finally all near-Moon with lunar surface nodes are combined together (all color coded). The bottom two plots in each figure show two different iterations of the NewmanGirvan algorithm with the resulting clusters colored differently. Clearly, there is no good match in either case. This means that clustering and linkage are designed in specific ways in mechanical systems, with characteristics and purpose that cannot necessarily be uncovered by traditional network analysis.

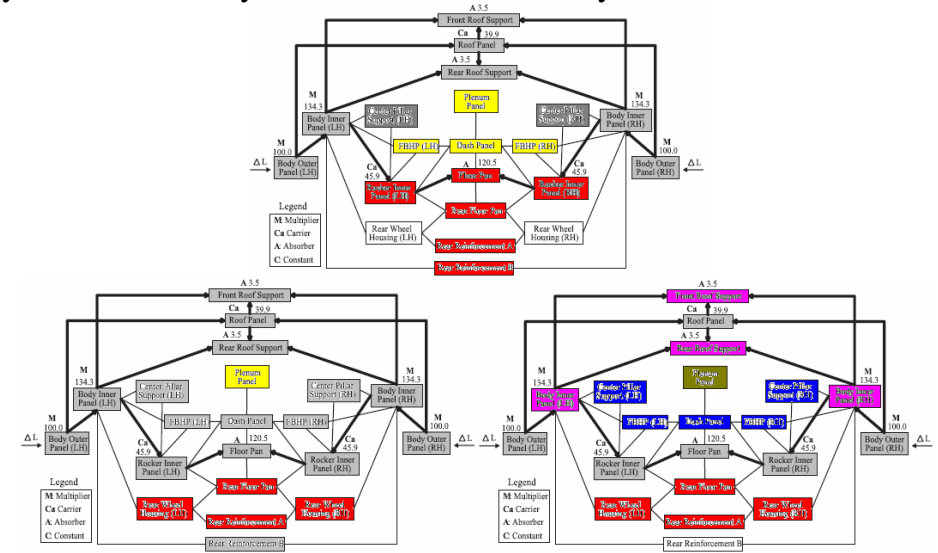

Figure 5: Modular structure of car component network: top 2 are Newman-Girvan splits, the bottom is the physically meaningful representation 


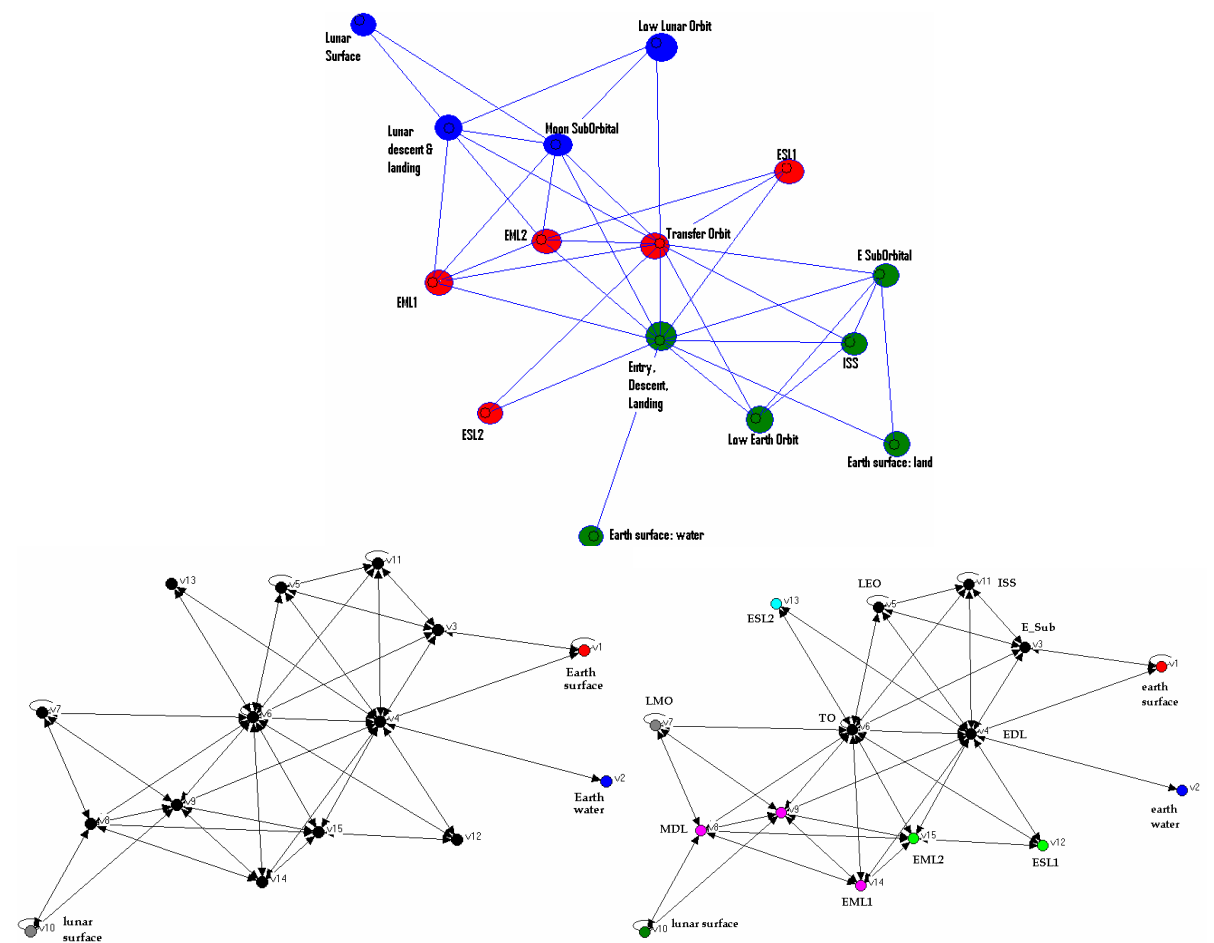

Figure 6: Spacenet modularized: constraining Newman-Girvan up to min 4 clusters gives three individual nodes as clusters, earth surface, earth water and lunar surface, which does have some meaning, as in final destination nodes, but nothing provides the expected near-Earth, near-Moon, and transfer trajectories.

Another focus in the network literature for system analysis is node centrality. Various measures of centrality exist, the simplest of which are degree centrality and betweenness centrality (also closeness, eigen-centrality, etc). Degree distributions and node significance have been widely employed in understanding structure and dynamics of social networks. To see how these considerations relate to the variety of systems we explore, we look at adjacency matrix dot alignments. That means aligning nodes against nodes and drawing dots if a link between two of them exists. Depending on the node order in this plot, interesting or no patterns can emerge. Figure 7 shows the matrix alignments for the systems in this study. The matrices are ordered by design (as recorded), by increasing nodal degree, increasing betweenness and then increasing eigen-centrality. It is evident right away that for a different system, different metrics contain the important structural information. For example, the design plot and the degree plot do not tell much about the MAPK pathway. The betweenness plot on the other hand shows obvious structure. In the case of the technological networks, it can be argued that the design plot (which is probably recorded component-wise) contains the most information. In any case, our aim was to show that traditional network metrics and structural considerations cannot be applied to all systems equal. 
a)
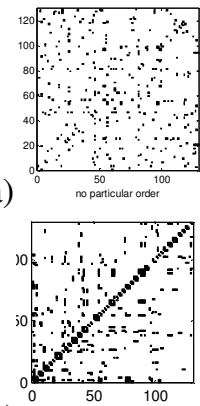

b)

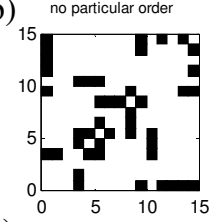

c)

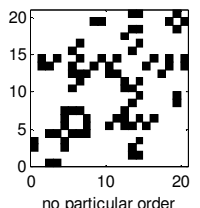

d)

e)

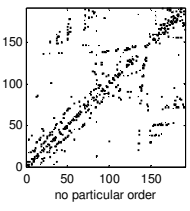

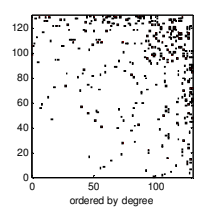

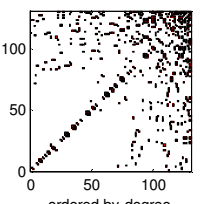

ordered by degree
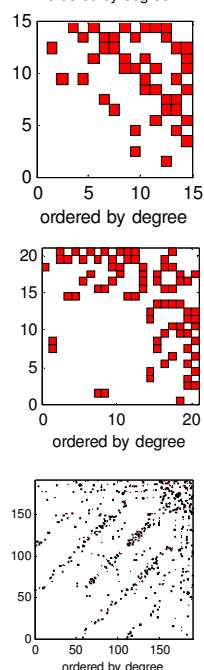
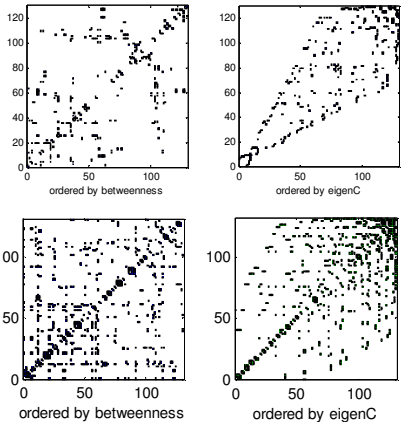

ordered by betweenness
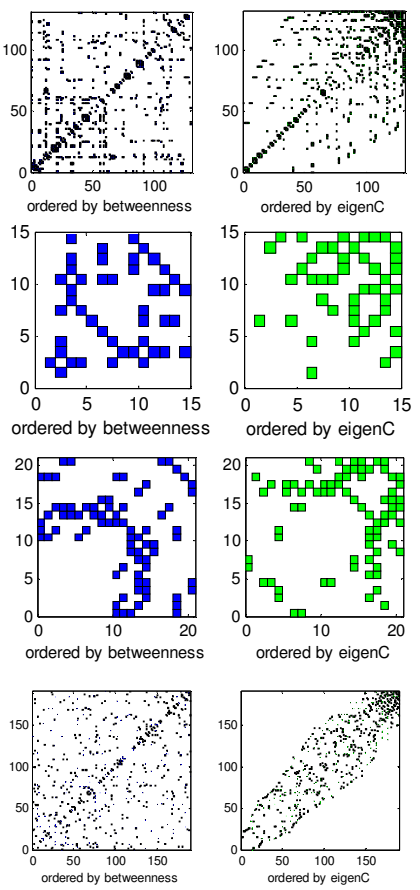

Figure 7: Adjacency matrix dot-alignments, node ordering by (design), increasing degree, increasing betweenness, and eigen-centrality; a) MAPK human pathway, b) ESD journal faculty network, c) spacenet model, d) car component frame network, e) Tokyo subway.

In engineering systems, compared to node analysis, path finding is more important. This is because parts and components are more likely to be well-defined, engineered and understood, whereas the whole system might have an emergent behavior. Often an engineer cares about change propagation (stress, cracks, innovation) or in mission analysis, optimal path finding, given thousands of options. Thus understanding the network relationships in the system has an inherently different purpose. Technological systems have to be operated, often fulfill a single purpose or have some finite functional spectrum. The main difference is that they do not emerge, but are designed to operate. So any structural analysis, should at a minimum uncover the schematics of the designer. As we saw, simple network models do not represent the structure of complex engineering systems very well. Our interest is less in structure, but more so in dynamics, operation at various levels and eventually growth, expansion or shrinking of the system. A car frame propagates vibration and has to be integral, yet somewhat modular to withstand design changes without complete replacement. A space 
transportation network only serves to provide route optimization for mission planning. To serve a design challenge right, such a model has to convey the right level of information - where can one get to physically and how much effort does it take? While there is politics involved in design decisions, one can only attempts to make an objective decision, as many design teams have done.

Table 1: Simple graph statistical measures for four different systems: a journal publication network, a car frame component network, a space transportation model and the MAPK reference pathway.

\begin{tabular}{|c|c|c|c|c|c|}
\hline & $\begin{array}{c}\text { MAPK } \\
\text { Reference } \\
\text { pathway } \\
\text { network }\end{array}$ & $\begin{array}{c}\text { Journal } \\
\text { publication } \\
\text { network for } \\
\text { MIT ESD }\end{array}$ & $\begin{array}{c}\text { Space } \\
\text { Transportation } \\
\text { Net }\end{array}$ & $\begin{array}{c}\text { Car } \\
\text { Component } \\
\text { Network }\end{array}$ & $\begin{array}{c}\text { Tokyo } \\
\text { Subway }\end{array}$ \\
\hline $\begin{array}{l}\text { Network } \\
\text { type }\end{array}$ & Biological & Social & Technological & Technological & $\begin{array}{c}\text { Technologi } \\
\text { cal }\end{array}$ \\
\hline $\mathbf{N}$ & 143 & 196 & 15 & 21 & 191 \\
\hline $\mathbf{M}$ & 176 & 547 & 48 & 39 & 297 \\
\hline $\mathbf{m} / \mathbf{n}$ & 1.2308 & 2.791 & 3.2 & 1.8571 & 1.555 \\
\hline Directed? & $\begin{array}{c}\text { No (as } \\
\text { modeled) }\end{array}$ & No & Yes & No & No \\
\hline $\begin{array}{l}\text { Hybrid } \\
\text { nodes? }\end{array}$ & Yes & No & Yes & Yes & No \\
\hline $\begin{array}{l}\text { Hybrid } \\
\text { links? }\end{array}$ & Yes & No & Yes & No & No \\
\hline Dynamic? & $\begin{array}{l}\text { Static (as } \\
\text { modeled) }\end{array}$ & $\begin{array}{l}\text { Static (as } \\
\text { modeled) }\end{array}$ & $\begin{array}{l}\text { Dynamic (as } \\
\text { modeled) }\end{array}$ & Static & Static \\
\hline $\begin{array}{l}\text { Number of } \\
\text { conn. } \\
\text { components }\end{array}$ & $15,\{\mathrm{GC}-126\}$ & $16\{\mathrm{GC}-131\}$ & In: $2\{14,1\}$ & 1 & 1 \\
\hline $\begin{array}{l}\{\max , \\
\text { mean, min } \\
\text { \}degree }\end{array}$ & $\{15,2.831,1\}$ & $\{33,6.565,1\}$ & $\begin{array}{c}\text { Tot }\{16,6.4,1\} \text {, } \\
\text { in }\{8,3.2,1\}, \\
\text { out: }\{10,3.2,0\}\end{array}$ & $\{8,3.7143,1\}$ & $\{9,3.11,1\}$ \\
\hline $\begin{array}{l}\text { Most } \\
\text { connected } \\
\text { node }\end{array}$ & p38 $(\operatorname{deg}=15)$ & $\begin{array}{l}\text { Management } \\
\text { Science }\end{array}$ & $\begin{array}{l}\text { Tot: TO, in: } 5, \\
\text { out: TO }\end{array}$ & $\begin{array}{c}\text { Body Inner } \\
\text { Panel (left and } \\
\text { right) }\end{array}$ & 51 \\
\hline $\begin{array}{l}\text { Degree } \\
\text { correlation }\end{array}$ & -0.306 & -0.0229 & $\begin{array}{c}0.0402(\mathrm{GC} \\
0.2011)\end{array}$ & -0.5255 & 0.016 \\
\hline $\begin{array}{l}\{\text { max }, \text { mean } \\
, \text { min\} betw }\end{array}$ & $\begin{array}{c}\{4639,1035.5 \\
384\} \\
(32.44 \text { times } n)\end{array}$ & $\begin{array}{c}\{1029,375, \\
228\}(5.25 \\
\text { times } \mathrm{n})\end{array}$ & & $\begin{array}{c}\{89,48.714 \\
32\} \\
(4.23 \text { times } n)\end{array}$ & $\begin{array}{c}\{940, \\
420.288, \\
289\}\end{array}$ \\
\hline $\begin{array}{l}\text { Clustering } \\
\text { coefficient }\end{array}$ & 0.008 & 0.807 (GC) & $\begin{array}{c}0.1389 \text {, in: } \\
0.1792\end{array}$ & 0.2512 & $\begin{array}{l}0.081 \\
0.059\end{array}$ \\
\hline $\begin{array}{l}\text { Number of } \\
\text { triangle } \\
\text { loops }\end{array}$ & 3 & 697 & 10 & 10 & 23 \\
\hline $\begin{array}{l}\text { Number of } \\
\text { rectangular } \\
\text { loops }\end{array}$ & 400 & 76 & 0 & 52 & 148 \\
\hline $\begin{array}{l}\text { Network } \\
\text { diameter }\end{array}$ & $17(11 \% \mathrm{n})$ & $6(3 \% \mathrm{n})$ & $7(46 \% \mathrm{n})$ & $5(23 \% n)$ & 14 \\
\hline $\begin{array}{l}\text { Mean path } \\
\text { length }\end{array}$ & $\begin{array}{c}6.454(37 \% \mathrm{~d}, \\
4 \% \mathrm{n})\end{array}$ & $\begin{array}{c}3.3(55 \% \mathrm{~d}, \\
1 \% \mathrm{n})\end{array}$ & $\begin{array}{c}2.5561(36 \% \mathrm{~d} \\
17 \% \mathrm{n})\end{array}$ & $\begin{array}{c}2.3333(46 \% \mathrm{~d}, \\
11 \% \mathrm{n})\end{array}$ & $\begin{array}{c}6.194 \\
(44.2 \% \mathrm{~d}, \\
3.2 \% \mathrm{n})\end{array}$ \\
\hline $\begin{array}{l}\text { Number of } \\
\text { communities } \\
\text { (clusters) }\end{array}$ & $\begin{array}{l}\text { Newman- } \\
\text { Girvan: } 4\end{array}$ & 7 & $\begin{array}{c}\text { Phys: } 3 \text {, } \\
\text { Newman-Girvan: } \\
3 \text { or } 8\end{array}$ & $\begin{array}{c}\text { Phys: } 3 \text {, } \\
\text { Newman- } \\
\text { Girvan }>5\end{array}$ & 13-14 \\
\hline
\end{tabular}




\begin{tabular}{|c|c|c|c|c|c|}
\hline & & & & meaningful & \\
\hline $\begin{array}{l}\text { Performance } \\
\text { metrics }\end{array}$ & $\begin{array}{c}\text { Effective } \\
\text { response to cell } \\
\text { stimuli }\end{array}$ & $\begin{array}{c}\text { Team work? } \\
\text { Collective } \\
\text { publishing } \\
\text { success? }\end{array}$ & $\begin{array}{l}\text { Overall mission } \\
\text { success - } \\
\text { easiness of } \\
\text { getting from one } \\
\text { location to } \\
\text { another }\end{array}$ & $\begin{array}{l}\text { Ability to } \\
\text { handle } \\
\text { vibration, } \\
\text { shocks; } \\
\text { flexibility in } \\
\text { accepting } \\
\text { change }\end{array}$ & $\begin{array}{l}\text { Efficient } \\
\text { transp. } \\
\text { service for } \\
\text { passengers } \\
\text { - travel } \\
\text { time and } \\
\text { connection }\end{array}$ \\
\hline Cost metrics & $\ldots$ & & $\begin{array}{c}\text { Mission cost } \\
\text { (fuel, life } \\
\text { support, power) }\end{array}$ & Production cost & $\begin{array}{c}\text { Infrastruct } \\
\text { ure cost }\end{array}$ \\
\hline
\end{tabular}

\subsection{Augmented Network Model}

The key characteristics of an augmented network model are that i) it contains more information than an edge list, ii) every model is domain-specific and iii) network theoretical tools can be used for global analysis, especially in pointing to interesting areas for research.

\section{Node refinement}

As argued in previous sections, understanding and designing an engineering system required domain knowledge and models augmented with engineering detail. We have tested a simple methodology for such proposed analysis using the space transportation model, described in Section 1.2.4. Nodes have three types (surface, sequence and orbit) and are described with relevant names, types, geometrical coordinates, set of states, internal node time counter, associated delta $\mathrm{V}$ (change in velocity magnitude), if any.

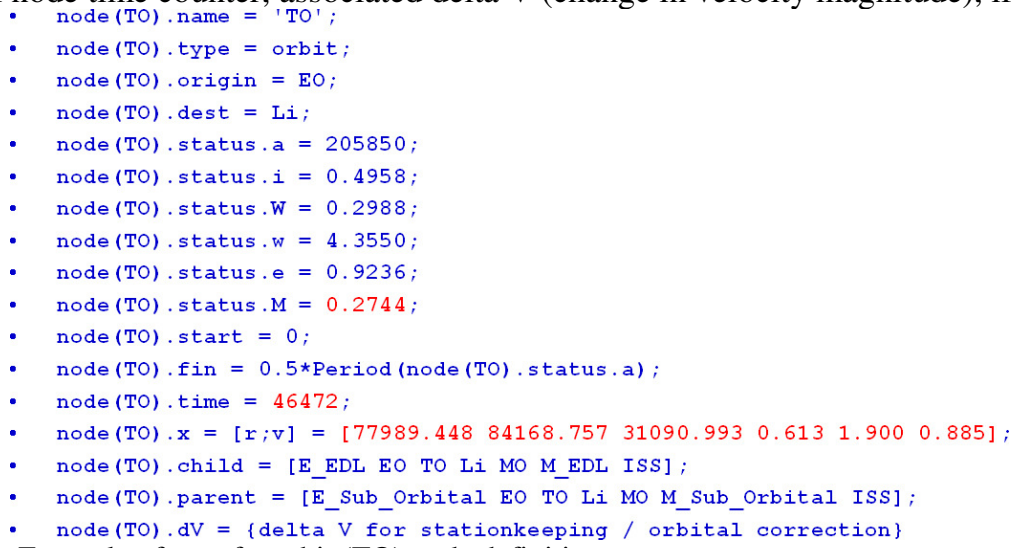

Figure 8: Example of transfer orbit (TO) node definition.

\section{Edge refinement}

The next step is edge refinement. Given the model used, state-space, physical, geometrical, links can have different meaning. An acquaintance link can be described simply as a node pair, while a protein interaction edge can be augmented by environmental conditions under which interaction was detected, frequency of interaction, activation or inhibition and so on. In the case of the spacenet model, a link has associated delta $\mathrm{V}$ (fuel burn) and associated type (describing the state transition). 
For example, the TMI link described in Figure 8, connects an orbit node to another orbit node, hence its type is orb2orb.

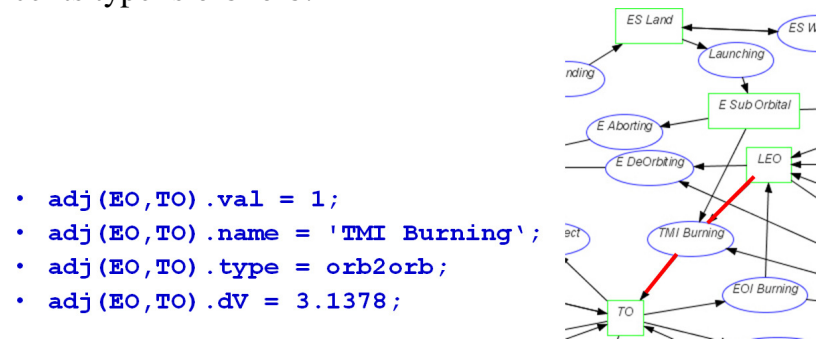

Figure 9: Translunar injection link example. It is specified in an adjacency matrix, as a link between EO (Earth orbit) and TO (transfer orbit)

\section{Rules of Dynamics}

The use of more detailed, design-relevant node and link description is to be able to encode rules of dynamics. For example, a link might be temporal, that is, it exists, but not at all times. There is a tight launch window from Earth orbit to lunar orbit, and then the lunar surface, that depends on the desired landing location. In general, the rules of dynamics describe what transitions (links) are possible under what conditions.

\section{Physical Simulation}

The system or parts of it can be simulated with given initial conditions or for a given purpose. The output, such as evaluating a pathway, can be linked to another set of models for dedicated component design for example.

\section{Network feedback: inferring centrality and meaningful paths from} network analysis for further inspection

After the physical simulation and a better understanding of the system, this process can be iterated with investigating different network perspectives of the entire model.

\subsection{Conclusion}

These are apparently systems of different domains, size and level of heterogeneity. The relevance of network modeling is variable in each case, because of the domain biases and data collection problems, as well as the implications for analysis and design. For example, social network studies have long been employed to study community structure, functional clusters and prominent nodes. Network structure and topology studies are fairly straightforward to do with homogeneous networks. In biological and technological systems domain knowledge becomes essential. We discussed examples of technological systems where structure is not simple to detect, and which cannot be modeled with homogeneous models. Finally, we present an augmented methodology for engineering design. Our model has been applied to do design in the case of the space transportation network [Bounova 2006]. 
Augmented Network Model for

Engineering System Design

\section{References}

Bounova et al, Selection and Technology Evaluation of Moon/Mars Transportation Architectures, Space 2005

Simmons et al. Mission Mode Architecture Generation for Moon-Mars Exploration Using an Executable Meta-Language, Space 2005

Hybrid Control Systems Laboratory, Stanford

KEGG database, source: http://www.genome.jp/kegg/pathway.html

Suh et al, Design for Flexibility: Performance and Economic Optimization of Product Platform Components, 10th AIAA/ISSMO Multidisciplinary Analysis and Optimization Conference, 2004

Newman , M. E. J., The structure and function of complex networks , SIAM Review 45, 167-256 (2003)

Whitney, D., Connectivity Limits of Mechanical Assemblies Modeled as Networks, ESD Working Paper Series, 2005

Bounova et al, Space Transportation Network Model for Rapid Lunar Architectures Analysis, Space Transportation Symposium, $57^{\text {th }}$ International Astronautical Congress, 2006 